Submission ID: 43840

\title{
Modern Seismic Field Acquisition
}

\section{M.B. Shneerson* (RPE "Special Geophysical Data Systems" Ltd.)}

\section{SUMMARY}

Seismic field acquisition play a great role in receiving good materials in seismic prospecting. The latest improvements in seismic acquisition is possible to read in materials of annual geophysical conventions. We used this materials to inform our specialists about news in field technologist. It is the aim of our report. We note next directions in modern field seismic acquisition: - theoretical and experimental investigations in seismic wave generation; - development of field vibroseis equipment. We demonstrate the high geological effectiveness of modern seismic field acquisition. 


\title{
Современные полевые технологии наземной сейсморазведки
}

\author{
М.Б. Шнеерсон (РГУ нефти и газа (НИУ) имени И.М. Губкина)
}

\section{Введение}

Полевые технологии наземной сейсморазведки непрерывно развиваются и совершенствуются, обеспечивая получение качественных материалов во все более сложных и разнообразных сейсмогеологических условиях. Наиболее полно вопросы технологии полевых сейсморазведочных работ находят освещение в докладах на ежегодных международных геофизических конференциях EAGE и SEG. Обзор этих материалов позволяет получить определенное представление о направлении и содержании проводимых работ, что представляет для нас несомненный интерес. Это и определило тематику и содержание доклада, которые основываются на материалах геофизических конференций 2015 и 2016 гг по технологии и методике полевых сейсморазведочных работ. Анализ содержания докладов позволяет сгруппировать их по следующим направлениям: - теоретические и экспериментальные исследования по возбуждению колебаний в вибрационной сейсморазведке; - технические средства возбуждения; эффективность современной технологии сейсморазведочных работ; и - адаптация методики полевых наблюдений к задачам и условиям работ.

\section{1. Теоретические и экспериментальные работы по возбуждению колебаний в вибрационной сейсморазведке}

Повышение эффективности возбуждения колебаний в вибрационной сейсморазведки остается важной задачей, и ей посвящено несколько работ. Наиболее значимые из них - это усложнение модели грунта (Wei, Pan, 2015) и учет распределения нагрузок под плитой вибратора (Dean, Vermeer, Laucock, 2015). В первой работе предложена усложненная модель системы вибраторгрунт, в которой присоединенный объем грунта представляется в виде последовательнопараллельно соединенных массы, упругости и затухания, которые, в совокупности оказывают искажающее влияние на возбуждаемые вибратором колебания. Для уменьшения этого влияния предложено фильтровать управляющий сигнал и с ним коррелировать исходные вибротрассы. На рис.1 приведены временные разрезы, полученные с нефильтрованным и фильтрованным сигналами, которые подтверждают целесообразность применения усложненной модели вибраторгрунт.

Во второй работе на основании измерений давления под плитой вибратора установлено, что его распределение носит существенно более сложный, чем ранее предполагалось, характер. И это оказывает отрицательное влияние на стабильность возбуждаемых колебаний. В наибольшей степени на характер распределения нагрузок под плитой вибратора оказывают ее жесткость и контакт с грунтом. Не остаются постоянными с глубиной и фазовые соотношения между излучаемым сигналом и распространяющейся волной, что осложняет контроль за работой вибратора. На основании полученных результатов авторы указывают на необходимость более детального и углубленного изучения процессов взаимодействия плиты вибратора с грунтом и распространения возбуждаемых волн в ближней от плиты вибратора зоне, а также на оптимизацию способов передачи нагрузок среде.

\section{2. Технические средства для возбуждения колебаний}

Возбуждению колебаний в вибрационной сейсморазведке посвящено значительное число работ. Наиболее значимые из них связаны с расширением спектрального состава возбуждаемых колебаний, опробованием псевдослучайных и кодовых управляющих сигналов, а также с оценкой работы вибраторов в вибрационном и импульсном режимах. Расширение спектрального состава возбуждаемых волн предлагается решать на основе модернизации существующих конструкций вибраторов, создания новых моделей излучателей, а также путем перехода на возбуждения 
небольших по диапазону частотных интервалов, которые в сумме перекрывают желаемую полосу частот. Наиболее интересны работы по новым моделям вибраторов. Описание одной из них для работ ВСП приведен в (Wei, Pan, 2015). Для расширения полосы возбуждаемых колебаний в сторону низких и высоких частот был разработан, изготовлен и успешно опробован относительно маломощный, широкополосный вибратор (усилие 115,65 N, ход поршня 10,16 см, максимальная частота 400 Гц, плита повышенной жесткости). Скважинные измерения показали, что на глубине 824 м наблюдался сигнал полосой от 2 до 300 Гц, а на глубине 2288 м были выделены колебания с максимальной частотой 150 Гц.

Определенный интерес представляет идея разделения частотного диапазона возбуждаемых колебаний на отдельные интервалы, в пределах которых колебания возбуждаются группами или одиночными вибраторами (Bogaini, 2016). Расстояния между источниками выбираются с условием обеспечения синфазного сложения волн различного частотного состава. По мнению авторов работа вибраторов в узкой полосе частот обеспечивает получение качественных записей, делает возможным повышение производительности работ и улучшение условий эксплуатации вибраторов

Значимым в настоящее время направлением развития вибрационной .сейсморазведки является использование псевдослучайных управляющих сигналов и $\mathrm{M}$ последовательностей. Ранее псевдослучайные сигналы применялись при проведении работ вблизи зданий и промышленных сооружений для исключения возможности из разрушения. В последнее время эти сигналы начали использоваться для исключения взаимного влияния волн при работе нескольких сейсмических партий в пределах одной площади, а также для получения низкочастотных записей от высокочастотного источника, генерирующего полупериодные псевдослучайные сигналы (Nakamura, Takanashi, 2016). В докладе по этому направлению приведены результаты теоретических и экспериментальных работ по применению постоянных по частоте управляющих сигналов (свипов) с псевдо-случайными временными изменениями амплитуд волн. Из теории следует, что автокорреляционные функции таких сигналов по форме близки к дельта-функции, что делает возможным их использование для получения широко-полосных сейсмических записей. Рассматриваемые экспериментальные работы предусматривали проведение межскважинных наблюдений в двух неглубоких скважинах глубиной 50 м. Волны возбуждались пьезоэлектрическим вибратор в полосе частот $100-2100$ Гц при работах по стандартной технологии и на постоянной частоте 2000 Гц - по новой. Сравнение прокоррелированных записей, полученных с псевдослучайными и стандартными управляющими сигналами показало, что первые более широкополосны и характеризуются существенно меньшим уровнем корреляционных шумов.

\section{3 Эффективность современной технологии сейсморазведочных работ}

Повышение геологической эффективности сейсморазведочных работ было и остается одним из важных направлений развития метода, и оно нашло широкое освещение в материалах конференций. В докладах это направление работ связывают с широко-полосным возбуждением (приемом) колебаний и применением высоко-плотных, широко-азимутальных систем наблюдений, в том числе с бескабельными, нодальными приемо-регистрирующими устройствами. В качестве примера на рис. 2 приведен пример существенного повышения качества материалов на основе современной широко-полосной и высоко-плотной технологии работ. (Zhao, Wang, Zhang, 2016).

\section{4. Адаптация методики полевых наблюдений к задачам и условиям работ}

В этот раздел вошли работы, иллюстрирующие возможность высокой степени адаптации методики полевых работ к сложным условиям их проведения. Практика показывает, что практически для любого района может быть предложена и реализована технология полевых наблюдений, обеспечивающая получение качественных исходных материалов. В качестве примера в (Jianjun, Jie, Yong, 2015) представлены результаты съемки 3D в пустынных районах 


\section{EAGE}

Западного Китая, которые характеризуются следующими тремя особенностями: - 1) закрытость региона для прослеживания глубоких отражений, - 2) высокий уровень кратных волн и - 3) латеральные изменения значительных по мощности зон выветривания. В этих условиях только высоко-плотные, широко-азимутальные системы наблюдений (бин 25м х 25м, кратность - 1350 , аспектное отношение 0,9 и плотность трасс 2160000 на км²) в сочетании с методикой «скользящего свипа» (время смещения $10 \mathrm{c}$ ), низко-частотным возбуждением колебаний (группирование 2-х вибраторов, свип 1,5 - 84 Гц, длительность 20с ) и тщательной коррекцией статических поправок обеспечили получение качественных материалов. Кроме того, для лучшего контакта плит источников колебаний и сейсмоприемников работы проводились при отсутствии снежного покрова. На рис. 3 приведен пример, иллюстрирующий эффективность примененных методических приемов.

Снижение цены на нефть привело к сокращению средств, выделяемых на сейсморазведку. В этой связи в докладе (Tellier, Poste) рассмотрены технические решения, позволяющие снизить стоимость работ с вибрационными источниками колебаний. По мнению авторов это: повышение производительности работ за счет уменьшения длительности низкочастотных нелинейных управляющих сигналов и модернизации существующих вибраторов путем увеличения «хода поршня» и инерционной массы вибровозбудителя и совершенствования гидравлической системы вибратора, а также путем уменьшения непроизводительных затрат времени на заправку и дозаправку излучателей и подготовку их к работе.

В заключении отметим, что по каждому из отмеченных направлений работ будут приведены материалы, позволяющие оценить полученные результаты.

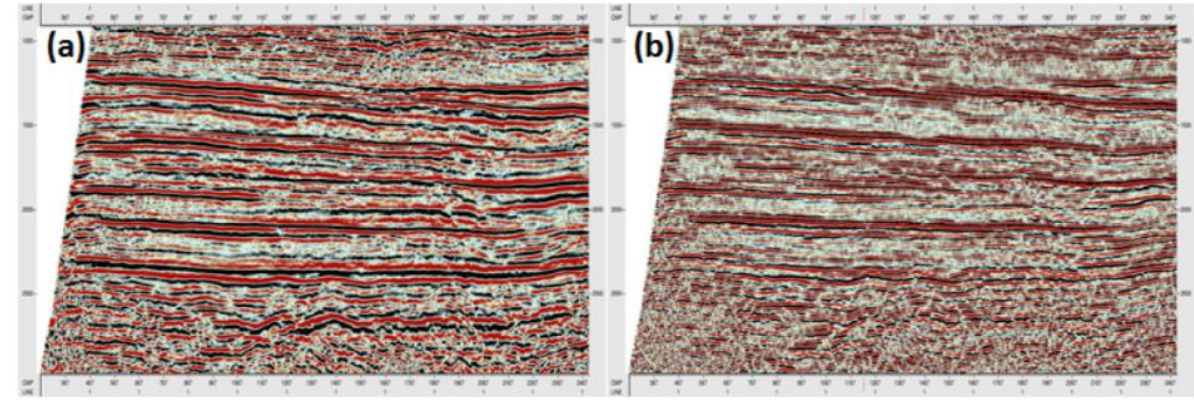

Figure 4 2D section image comparison. (a) Geophone data correlated with the pilot sweep. (b) Geophone data correlated with the pilot sweep filtered by the complex vibrator-ground model.

Рисунок 1 Временные разрезы, полученные с нефильтрованным (a) и фильтрованным (b) сигналами

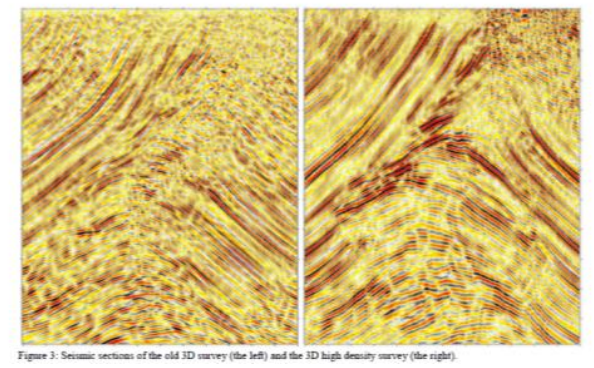

Рисунок 2 Фрагменты временных разрезов, полученных по «старой» слева и «новой» справа технологиям 

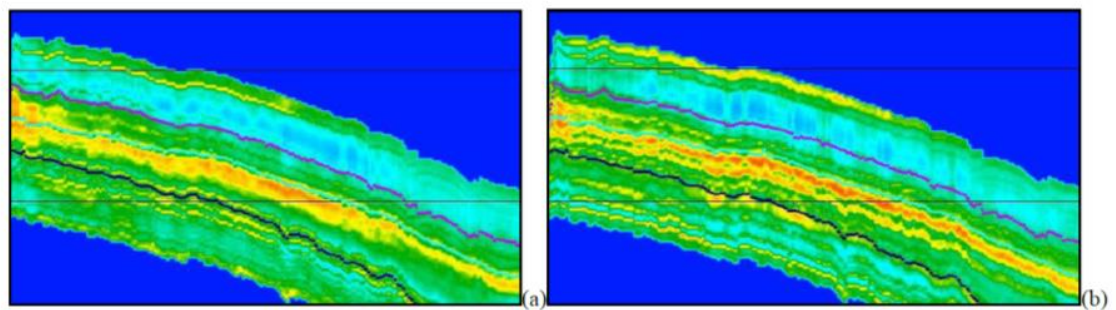

Figure 5 P-wave impedance inversion of conventional exploration (a) and broadband exploration (b).

Рисунок 3 Результаты инверсии по материалам стандартной (a) и широкополосной (b) сейсморазведки

\section{Выводы}

Современные технологии наземной сейсморазведки являются достаточно эффективным средством изучения строения глубинных недр, обеспечивая получение качественных первичных материалов в различных сейсмо-геологических условиях. Перспективы развития технологии связаны с применением используемых методических и технических средств, а также с созданием новых, более эффективных технологий, базирующихся на решении теоретических и технических задач по оптимизации условий и способов возбуждения колебаний.

\section{Литература / References}

Bogaini, C.,2016, 2016, Shared low frequency vibroseis acquisition, Expanded Abstracts, Vena Dean,T., Vermeer, P.,Laycock, M.,Tulett, J., 2015,The complexity of vibrator baseplate-ground interaction measured with a thin-film pressure pad and downhole tool, Expanded Abstracts, Madrid Jianjun, H., Jie,Y., Yong, G.,2015, A 3-D acquisition case surveying strata underlying shielding zones in a large desert area, Expanded Abstracts, New Orlean

Nakamura, Y., Takanashi, M., Nakatsukasa, M., 2016, Low-frequency data acquisition from highfrequency source using half-cycle pseudorandom sweep, Expanded Abstracts, Vena

Tellier, N., Poste,J., 2016, Reducing the operational cost of seismic vibrators, Expanded Abstracts, Dallas

Wei, Z. Pan, Y., 2015, Improving vibroseis data quality with the vibrator-ground model, Expanded Abstracts, Madrid

Zhao, X., Wang, X., Zhang, R., 2016, Land broadband seismic exploration based on adaptive vibroseis, Expanded Abstracts, Dallas 